\title{
Microarchitecture Level Power and Thermal Simulation Considering Temperature Dependent Leakage Model
}

\author{
Weiping Liao Fei Li Lei He \\ Electrical Engineering Department \\ University of California at Los Angeles
}

\begin{abstract}
In this paper, we present power models with clock and temperature scaling, and develop the first of its type coupled thermal and power simulation with temperature-dependent leakage power model at micro-architecture level. We show that leakage energy and total energy can be different by up to $2.5 X$ and $2 X$ for temperatures between $90^{\circ} \mathrm{C}$ and $130^{\circ} \mathrm{C}$, respectively. Given such big energy variations, no power model at microarchitecture level is accurate without considering temperature dependent leakage models.
\end{abstract}

\section{Categories and Subject Descriptors}

B.7.2 [Integrated Circuits]: Simulation.

\section{General Terms}

Design.

\section{Keywords}

Leakage, thermal, simulation.

\section{INTRODUCTION}

A number of cycle accurate microarchitecture level power simulators $[1,2,3,4]$ have been developed for microarchitecture optimization. However, all these simulators consider only cycle-based power models without clock scaling, i.e., the dynamic and leakage energy is given per clock cycle, and the ratio between them is invariant with respect to the clock rate. This assumption of fixed leakage and dynamic energy ratio is no longer valid with clock scaling because for each clock cycle, the dynamic energy does not depend on clock period but the leakage energy does.

Temperature scaling is another important factor in power modeling. There is some limited study on the thermal modeling. [5] evaluates the thermal impact on future nanometer VLSI design from technology scaling point of view. [6] discusses the thermal performance for Intel's processors with

Permission to make digital or hard copies of all or part of this work for personal or classroom use is granted without fee provided that copies are not made or distributed for profit or commercial advantage and that copies bear this notice and the full citation on the first page. To copy otherwise, to republish, to post on servers or to redistribute to lists, requires prior specific permission and/or a fee.

ISLPED’03, August 25-27, 2003, Seoul, Korea.

Copyright 2003 ACM 1-58113-682-X/03/0008 ...\$5.00. emphasis on the package material and cooling mechanism. $T E M^{2} P^{2} E S T$ [3] is the first microarchitecture simulator with built-in thermal model. However, leakage power models in $[1,2,3,4]$ are independent of temperature, and may lead to severe estimation error given that leakage power is an exponential function of temperature.

In this paper, we present power models with clock and temperature scaling, and develop the coupled thermal and power simulator at microarchitecture level. With this simulator, we are able to accurately simulate the inter-dependence between the power and temperature, obtain accurate power and thermal profile, and evaluate microarchitecture level power and thermal management techniques. The rest of this paper is organized as follows: In Section 2, we propose our power models. In Section 3, we present our thermal model. In Section 4, we present the experiments on thermalsensitive energy simulations as well as the impact of clock gating. To the best of our knowledge, this is the first paper considering the inter-dependence between clock rates, system energy and temperatures at microarchitecture level.

\section{LEAKAGE POWER CALCULATION}

We define three power states same as [4]: (i) active mode, where a circuit performs an operation and dissipates both dynamic power $\left(P_{d}\right)$ and leakage power $\left(P_{s}\right)$. The sum of $P_{d}$ and $P_{s}$ is defined as active power $\left(P_{a}\right)$. (ii) standby mode, where a circuit is idle but ready to execute an operation, and dissipates only leakage power $\left(P_{s}\right)$. (iii) inactive mode, where a circuit is deactivated by power gating or other leakage reduction techniques, and dissipates a reduced leakage power defined as inactive power $\left(P_{i}\right)$. A circuit in the inactive mode needs non-negligible amount of time to wake up and then perform an useful operation.

In cycle accurate simulations, power is defined as the energy per clock cycle. Therefore, $P_{d}$ is equal to $\frac{1}{2} f_{s} C V^{2}$ where $C$ is the switching capacitance, $V$ is the supply voltage and $f_{s}$ is the switching factor per clock cycle. In essence, $P_{d}$ is the energy to finish a fixed number of operations during one cycle and is assumed to be independent of the clock rate in this paper. Consistently, $P_{s}$ is defined as $P_{s o} * t$ where $P_{s o}$ is leakage power per second and $t$ is the clock period. Same as $P_{s}, P_{i}$ is proportional to the clock period.

\subsection{Related Work on Leakage Modeling}

Microarchitecture level power simulators $[2,3,4]$ calculate leakage power by assuming a ratio between $P_{s}$ and $P_{d}$. For example, the ratio for logic circuits in [4] is decided by SPICE simulations on typical circuits. Furthermore, [4] also 
presented formula-based models for $P_{s}$ and $P_{i}$ in memory based units. The models in [2, 3, 4] are efficient for microarchitecture level simulations. But the ratio is independent of clock and temperature, and therefore it is not accurate.

Another high-level leakage power model is proposed in [7] with the following simple equation:

$$
P_{\text {so }}=V_{d d} \cdot N_{F E T} \cdot k_{\text {design }} \cdot \hat{I}_{\text {leakage }}
$$

where $V_{d d}$ is the supply voltage, $N_{F E T}$ is the number of transistors, $k_{\text {design }}$ is a design dependent parameter, and $\hat{I}_{\text {leakage }}$ is a technology dependent parameter. Both $k_{\text {design }}$ and $\hat{I}_{\text {leakage }}$ are different for different circuit types. However, there is no well-defined method to decide $k_{\text {design }}$ and

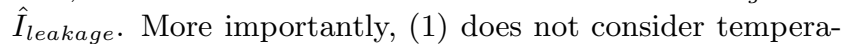
ture scaling.

\subsection{New Leakage Model for Logic}

In this paper, we propose a new leakage power model for logic circuits. As shown in (2), for a given circuit, the leakage power can be calculated as the product of gate number $\left(N_{\text {gate }}\right)$ and the average leakage current per gate $\left(I_{a v g}\right)$.

$$
P_{s o}=N_{\text {gate }} * I_{a v g} * V_{d d}
$$

$I_{\text {avg }}$ can be calculated by computing the average leakage current per gate for given $n$ circuits based on the gate-level estimation results. Because leakage current depends on different input vectors [8], we apply Genetic Algorithm for a few typical circuits, and obtain the $I_{a v g}$ for both maximum and minimum leakage currents. Figure 1 shows such $I_{a v g}$ calculation with respect to the number of circuits for both maximum and minimum leakage currents. It is easy to see that after the number of circuits exceeds 20, the value of $I_{\text {avg }}$ becomes very stable. Formula similar to (2) has been proposed in [9] which can consider statistic impact of the stack effect. However, no explicit method is proposed in [9] to calculate $I_{\text {avg }}$.

Also as shown in Figure 1, the average difference between maximum and minimum $I_{\text {avg }}$ is about $1.6 X$. To consider the worst-case leakage current, we always use the maximum leakage current in the rest of the paper. Furthermore, we propose the following temperature scaling for $I_{\text {avg }}$ considering the exponential relationship between the leakage power and temperature:

$$
I_{a v g}(T)=I_{s} * \exp \left(-\frac{\alpha}{T+273-\beta}\right)
$$

where $I_{s}$ is a constant value. The coefficients $\alpha$ and $\beta$ are decided by circuit designs. Values for $\alpha$ and $\beta$ as well as validation of (3) will be presented in Section 2.4.

\subsection{New Leakage Model for Memory Based Units}

Memory based units such as caches and register files are usually modeled by SRAM arrays. The formula-based leakage power model has been proposed in [4]. We propose the following temperature scaling based on the leakage model from [4].

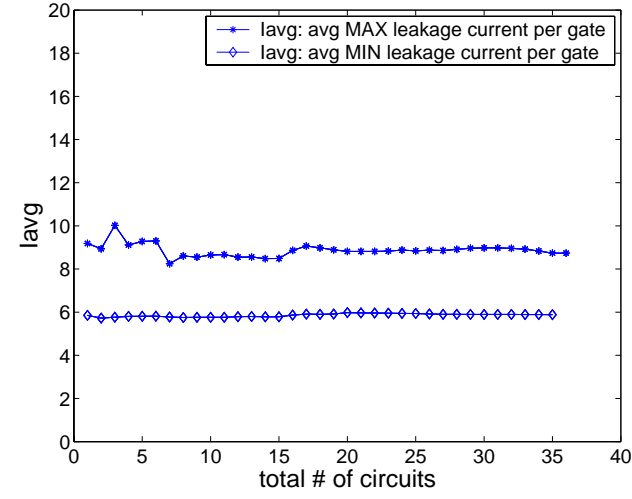

Figure 1: $I_{\text {avg }}$ calculation.

$$
\begin{aligned}
P_{\text {so }}= & P_{\text {circuits }}+P_{\text {cells }} \\
P_{\text {circuits }}= & (X * \text { words }+Y * \text { word_size }) \\
& * \exp \left(-\frac{\alpha}{T+273-\beta}\right) \\
P_{\text {cells }}= & (Z * \text { words*word_size }) \\
& * \exp \left(-\frac{\gamma}{T+273-\delta}\right)
\end{aligned}
$$

where $P_{\text {cells }}$ is the leakage power dissipated by SRAM memory cells and $P_{\text {circuits }}$ is the power generated by the circuits such as wordline drivers and precharge transistors, etc. $P_{\text {cells }}$ is proportional to the number of SRAM memory cells. $\alpha$ and $\beta$ in (5) are the same as those in (3), while $X, Y$, $Z, \gamma$ and $\delta$ in (5) and (6) are coefficients decided by circuit designs. Values for $X, Y, Z, \gamma$ and $\delta$ as well as validation of (5) and (6) will be presented in Section 2.4.

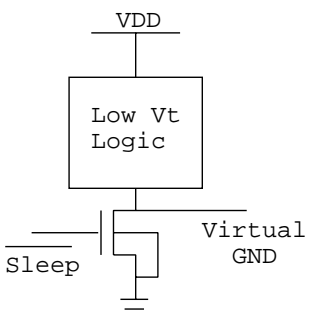

(A)

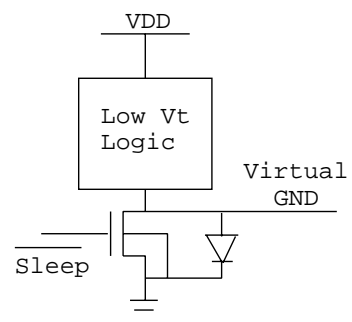

(B)
Figure 2: Power gating techniques: (a) MTCMOS and (b) VRC.

\subsection{Consideration of Leakage Reduction}

For both logic circuits and memory based unit, we can easily extend our leakage power model for leakage reduction techniques. For example, power gating techniques such as MTCMOS and VRC are used in [4]. Figure 2 shows the schematics for MTCMOS and VRC. In MTCMOS, a sleep transistor is inserted between the circuits and GND. When the sleep transistor is turned off, there is no power supply to the logic circuits. In VRC, a diode is inserted parallel with 
the sleep transistor and maintains the voltage level that is needed to keep the logic states in the circuits. VRC should be used for memory based units such as caches and TLBs for data retention. In these cases, the leakage power formulas still have the same format as we shown above, although the coefficients are different.

We collect the power consumption for different types of circuits at a few temperature levels by SPICE simulations. We then obtain the coefficients in (3) - (6) by curve fitting. Table 1 summarizes the coefficients for ITRS $100 \mathrm{~nm}$ technology we used, and Table 2 compares our high-level leakage power estimation for logic circuits and SRAM arrays with SPICE simulations in ITRS 100nm technology. The difference between our formulas and SPICE simulation is less than $6 \%$ without power gating, and is less than $15 \%$ with power gating. Note that with the presence of sleep transistors, the leakage power value is smaller due to the stack effect. For example, as shown in Table $2,13.88 \%$ difference of $P_{\text {so }}$ for a $128 \times 32$ SRAM array with power gating is about $0.035 \mathrm{~mW}$, which is equal to $11.5 \%$ of $P_{\text {so }}$ without power gating.

\begin{tabular}{|c|c|c|c|}
\hline \multicolumn{2}{|c|}{} & $\begin{array}{c}\text { With } \\
\text { power gating }\end{array}$ & $\begin{array}{c}\text { Without } \\
\text { power gating }\end{array}$ \\
\hline \multirow{3}{*}{$\begin{array}{c}\text { Logic } \\
\text { circuits }\end{array}$} & $X$ & $2.63 \mathrm{e}-6$ & $3.89 \mathrm{e}-4$ \\
\cline { 2 - 4 } & $Y$ & $2.49 \mathrm{e}-6$ & $1.24 \mathrm{e}-3$ \\
\cline { 2 - 4 } & $\alpha$ & 606.53 & 3040.11 \\
\cline { 2 - 4 } & $\beta$ & 192.02 & 23.37 \\
\hline \multirow{2}{*}{$\begin{array}{c}\text { Memory } \\
\text { based } \\
\text { units }\end{array}$} & $Z$ & $8.91 \mathrm{e}-5$ & $4.33 \mathrm{e}-4$ \\
\cline { 2 - 4 } & $\gamma$ & 2273.27 & 3168.35 \\
\cline { 2 - 4 } & $\delta$ & 3168.35 & 21.34 \\
\hline
\end{tabular}

Table 1: Coefficients in (3) - (6) for 100nm technology, where MTCMOS and VRC are the power gating techniques for logic and SRAM arrays, respectively.

\begin{tabular}{|c|c|c|c|c|}
\hline & $\mathrm{T}$ & \multicolumn{2}{|c|}{$I_{\text {avg }}$ or $P_{\text {so }}$} & abs. \\
\cline { 3 - 4 } circuit & $\left({ }^{\circ} \mathrm{C}\right)$ & formula & SPICE & err. $\%$ \\
\hline adder & 100 & 0.0249 & 0.0238 & 4.62 \\
\hline multiplier & 100 & 0.0228 & 0.0217 & 5.07 \\
\hline SRAM 128x32 & 110 & 0.298 & 0.304 & 1.97 \\
\hline SRAM 512x32 & 110 & 1.163 & 1.145 & 1.57 \\
\hline SRAM(VRC) 128x32 & 110 & 0.287 & 0.252 & 13.88 \\
\hline SRAM(VRC) 512x32 & 110 & 1.137 & 1.082 & 5.08 \\
\hline
\end{tabular}

Table 2: Comparison of $I_{a v g}$ for logic circuits and $P_{s o}$ for SRAM power model between our formula and SPICE simulation. The SRAM arrays are represented as "row number" $\mathrm{x}$ "column number". The units for $I_{a v g}$ and $P_{s o}$ are $\mathbf{u A}$ and $\mathbf{m W}$, respectively.

\section{TEMPERATURE CALCULATION}

We develop the thermal model based on conventional heat transfer theory [10]. The stable temperature in our thermal model can be calculated according to (7):

$$
T=T_{a}+R_{t} * P
$$

where $T$ is the stable temperature, $T_{a}$ is the ambient temperature, $P$ is the power consumption, $A$ is the area, and $R_{t}$ is the thermal resistance, which indicates the ability to remove heat to the ambient under the steady-state condition.

The dissipated power does not convert to temperature immediately because of the slow material response. Therefore, an exponential response is expected for the transient temperature. In our thermal model, we calculate the temperature change for any period from time $t_{1}$ to $t_{2}$ by (8) and (9):

$$
\begin{aligned}
& \Delta T_{+}=\left(T_{\text {max }}-T_{t 1}\right) * \exp \left(-\frac{t_{2}-t_{1}}{\tau_{\text {heat }}}\right) \\
& \Delta T_{-}=\left(T_{t 1}-T_{a}\right) * \exp \left(-\frac{t_{2}-t_{1}}{\tau_{\text {cool }}}\right)
\end{aligned}
$$

where $\Delta T_{+}\left(\Delta T_{-}\right)$are the increment(decrement) of the temperature from time $t_{1}$ to $t_{2}, T_{\max }$ is the maximum silicon temperature that the package supports, and $\tau_{\text {heat }}$ and $\tau_{\text {cool }}$ are heating and cooling time constants. The decision to increase or decrease the temperature at time $t_{2}$ is made by the following criterion:

Suppose the average power between $t_{1}$ and $t_{2}$ is $P_{\text {avg. If }}$ $T_{a}+R_{t} * P_{a v g}>T_{t_{1}}$, then the temperature increases; otherwise, the temperature decreases.

In our thermal model, we have two different modes with different granularities to calculate the temperature: (i) individual mode. We assume that there is no horizontal heat transfer between components, and calculate a temperature for each individual component. In general, the horizontal heat reduces the temperature gaps between components. So the individual mode essentially gives the upper bound of temperature gaps. (ii) universal mode, which is similar to the thermal model in $T E M^{2} P^{2} E S T$ [3]. We assume the whole processor as a single component with a uniform thermal characteristic and temperature. The universal mode gives the lower bound of the highest on-chip temperature.

\section{EXPERIMENT RESULTS}

Although our power and thermal models are applicable to any architecture, we study VLIW architecture in this paper. We integrate our thermal and power model into the PowerImpact [4] toolset. Instead of fixing the absolute thermal resistance value, we use the relative thermal resistance in our experiments. First, we select the thermal resistance of one integer unit as the unit thermal resistance, and further define the integer unit's area as the unit area. The thermal resistances for all other components are inversely proportional to their areas. Table 3 presents the microarchitecture configuration of the VLIW processors we study, and Table 4 summarizes the power consumption, the relative thermal resistances and the relative areas for all components in our system. We set the thermal time constants as $\tau_{\text {heat }}=\tau_{\text {cool }}=100 u s$, which are independent of component area.

\subsection{Chip Temperature}

In our experiments, we update temperatures after each time step $t_{s}$. We then update the power value with respect to new temperature for each $t_{s}$. Smaller $t_{s}$ gives a more accurate transient temperature analysis, e.g., $t_{s}=1$ cycle represents the cycle accurate temperature calculation. Figure 3 plots the transient temperature calculated under different $t_{s}$ shown as the percentages of the thermal time 


\begin{tabular}{|c|c|c|c|c|}
\hline Component & \multicolumn{4}{|c|}{ Configuration } \\
\hline Decode (MTCMOS) & \multicolumn{4}{|c|}{ 6-issue width } \\
\hline BTB & \multicolumn{4}{|c|}{512 entries 4-way associative, Two-level predictor } \\
\hline Register file & \multicolumn{4}{|c|}{128 integer and 128 floating-point registers with 64-bit data width } \\
\hline Memory & \multicolumn{4}{|c|}{ page size 4096 bytes, latency 30 cycles } \\
\hline Memory Bus & \multicolumn{4}{|c|}{8 bytes/cycle } \\
\hline ALU (MTCMOS) & Number & \multicolumn{3}{|r|}{ Latency } \\
\hline Integer & 4 & \multicolumn{3}{|c|}{1 cycle for add, 2 cycles for multiply and 15 cycles for division } \\
\hline Floating-point & 2 & \multicolumn{3}{|c|}{2 cycles for add/multiply, 15 cycles for division } \\
\hline Cache (VRC) & Size & Block size & Associativity & $\overline{\text { Policy }}$ \\
\hline L1 Instruction & $32 \mathrm{~KB}$ & 32 bytes & 2 & LRU \\
\hline L1 Data & $32 \mathrm{~KB}$ & 32 bytes & 4 & LRU \\
\hline L2 & $512 \mathrm{~KB}$ & 64 bytes & 8 & LRU \\
\hline
\end{tabular}

Table 3: System configuration for experiments.

\begin{tabular}{|c|c|c|c|c|c|}
\hline Component & $P_{a}$ & $P_{s}$ & $P_{i}$ & $R_{t}$ & Area \\
\hline BTB & 10.38 & 2.2385 & 0.0093 & 2048.2 & 0.49 \\
\hline I-L1 Cache & 90.28 & 28.18 & 0.027 & 277.9 & 4.28 \\
\hline D-L1 Cache & 90.78 & 28.28 & 0.027 & 275.4 & 4.30 \\
\hline L2 Cache & 666.53 & 437.07 & 0.287 & 30.1 & 31.61 \\
\hline Registers & 4.99 & 1.65 & 0.004 & 5012.4 & 0.24 \\
\hline Decode & 14.06 & 0.025 & 0.00032 & 1178.22 & 0.667 \\
\hline Integer ALU & 21.09 & 0.036 & 0.0005 & 1185.48 & 1.0 \\
\hline FPU & 42.18 & 0.073 & 0.001 & 592.74 & 2.0 \\
\hline
\end{tabular}

Table 4: Power consumption (in pJ/cycle), relative thermal resistance $R_{t}$ and relative areas for all components. We assume $125^{\circ} \mathrm{C}$ and $1 \mathrm{GHz}$ clock rate. The decode, integer ALU and FPU are only one unit among total six, four and two units.

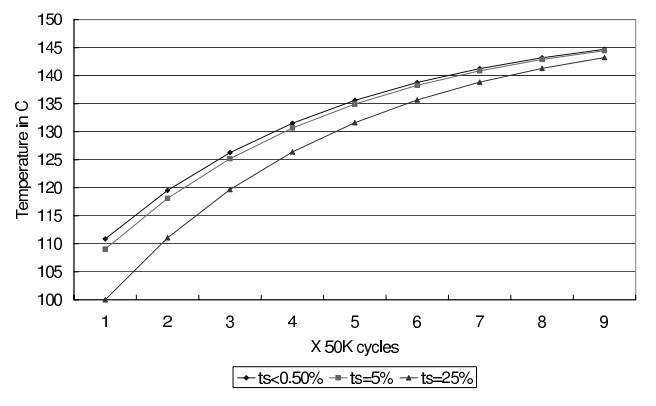

Figure 3: Temperature curve obtained by the universal mode for different time step $t_{s}$. The clock frequency is $2 \mathrm{GHz} .0 .5 \%, 5 \%$ and $25 \%$ of thermal time constant corresponds to 1000, 10000 and 50000 cycles, respectively. The benchmark is equake.

constant, where $0.5 \%$ of thermal time constants is equal to 1000 clock cycles for a $2 \mathrm{GHz}$ clock. When $t_{s} \leq 1000$ cycles (i.e. $0.5 \%$ of thermal constants), the temperatures are identical to those with $t_{s}=1$ cycle. Observable difference appears when $t_{s}$ is increased to $5 \%$ of the thermal constants and significant error is induced when $t_{s}=25 \%$ of the thermal constants. Furthermore, Table 5 presents the running time normalized with respect to that without temperature calculation. By setting $t_{s}$ to more than 100 cycles, we can reduce the running time by more than 5 times compared to $t_{s}=1$ cycle, and achieve virtually the same computation efficiency as the power simulation without temperature calculation. Since $0.5 \%$ of thermal constants are always more than 100 cycles for the clocks we study, and lead to negligible error on temperature calculation compared with the cycle accurate temperature calculation, we only update temperatures and power values after every period of $0.5 \%$ of the thermal time constants in the rest of the paper.

\begin{tabular}{|c|c|c|c|c|c|c|}
\hline$t_{s}$ (cycle) & N.T. & 1 & 10 & 100 & 500 & 1000 \\
\hline Running time & 1.0 & 5.66 & 1.49 & 1.05 & 1.03 & 1.01 \\
\hline
\end{tabular}

Table 5: Normalized running time for different temperature updating period. The N.T. means we do not have to update temperature and power during the whole simulation.

\subsection{Energy Consumption}

Figure 4 shows the experimental results for total energy consumption with different clocks. We assume there is no throttling, i.e., $P_{a}$ is dissipated in every cycle. We study two cases: one assumes a fixed temperatures, and another considers energy consumption with temperature dependence in both individual mode and universal mode. From Figure 4 we can see that by changing the temperature from $90^{\circ} \mathrm{C}$ to $130^{\circ} \mathrm{C}$, the total leakage energy can be changed by a factor of $2.5 X$, and the total energy is changed by up to $30 \%$. Figure 4 clearly shows that any study regarding to leakage energy is not accurate if the thermal issue is not considered. To consider temperature using methods in $[4,11]$, we may assume a fixed temperature appropriate for the processor and the environment, and then use circuit-extracted leakage values for that temperature. As shown in Figure 4, how to decide the appropriate temperature is of paramount importance for accurate energy estimation, and it is an open problem in the literature. Given the dependence between temperature and leakage energy, our work actually presents an approach to select the appropriate temperature point. Note that we assume that dynamic energy is independent of clock. This assumption will be revisited in Section 5 .

Figure 4 also shows that the total leakage energy is re- 


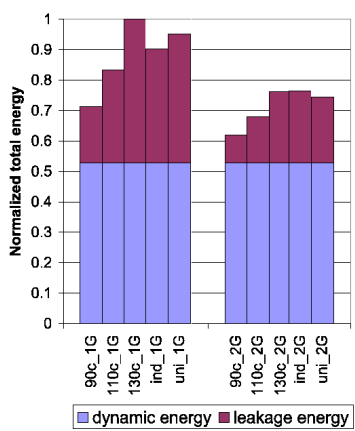

Figure 4: Energy consumption without any throttling. We study fixed temperatures of $90^{\circ} \mathbf{C}, 110^{\circ} \mathbf{C}$ and $130^{\circ} \mathbf{C}$, as well as the case with temperature dynamically updated. The prefix of "ind" and "uni" stand for the individual mode and universal mode, respectively. The benchmark is equake.

duced as the clock increases from $1 \mathrm{GHz}$ to $2 \mathrm{GHz}$, due to the reduced execution time for the given amount of work. This result implies that total leakage energy can be reduced by increasing system clock. However, the maximum temperature and maximum temperature gap constraints prevent us from increasing clock rate indefinitely. In our experiments, we assume the maximum allowable temperature is $130^{\circ} \mathrm{C}$ and the maximum temperature gap among components is $40^{\circ} \mathrm{C}$. We use the individual mode to calculate the maximum temperature and the maximum temperature gap, where the maximum temperature is set as the largest temperature among all temperature. Table 6 shows the maximum system temperature and the maximum temperature gap without any throttling. We can see that the maximum clock with thermal constraints is about $1 \mathrm{GHz}$ when there is no throttling.

\begin{tabular}{|c|c|c|c|c|c|}
\hline \multirow{2}{*}{ Benchmark } & $\begin{array}{c}\text { Clock } \\
(\mathrm{Hz})\end{array}$ & $500 \mathrm{M}$ & $1 \mathrm{G}$ & $1.5 \mathrm{G}$ & $2 \mathrm{G}$ \\
\hline \multirow{2}{*}{ equake } & Max T & 118.59 & 128.07 & 137.71 & 149.75 \\
\cline { 2 - 6 } & Max Gap & 5.96 & 9.86 & 14.85 & 21.92 \\
\hline \multirow{2}{*}{ go } & Max T & 118.58 & 128.06 & 137.6 & 147.52 \\
\cline { 2 - 6 } & Max Gap & 3.76 & 6.8 & 14.51 & 22.1 \\
\hline \multirow{2}{*}{ ijpeg } & Max T & 118.6 & 128.07 & 137.7 & 144.67 \\
\cline { 2 - 6 } & Max Gap & 3.76 & 6.8 & 14.5 & 17.04 \\
\hline
\end{tabular}

Table 6: Maximum temperatures (Max T) and temperature gaps (Max Gap) among components for different clocks without any throttling. The unit for temperatures is ${ }^{\circ} \mathrm{C}$.

\subsection{Impact of Clock Gating}

Clock gating [12] is effective to reduce dynamic power by turning off the clock signal for idle components. It also reduces total leakage energy by lowering the temperature. In this section, we consider the ideal clock gating, i.e. no overhead to turn on and off the clock for a component, and present a quantitative study on the impact of clock gating.

In [4], the instructions are always assigned to preferred functional units first. This method makes the preferred units much busier, and therefore have much higher temperatures

\begin{tabular}{|c|c|c|c|c|c|c|}
\hline Benchmark & $\begin{array}{c}\text { Clock } \\
(\mathrm{Hz})\end{array}$ & $500 \mathrm{M}$ & $1 \mathrm{G}$ & $1.5 \mathrm{G}$ & $2 \mathrm{G}$ & $2.5 \mathrm{G}$ \\
\hline \multirow[t]{2}{*}{ equake } & $\underset{\mathrm{T}}{\mathrm{Max}}$ & $\begin{array}{c}11.4- \\
114.8\end{array}$ & $\begin{array}{c}113.5- \\
125\end{array}$ & $\begin{array}{c}115.6- \\
135.5\end{array}$ & $\begin{array}{c}117.5- \\
146.3\end{array}$ & $\begin{array}{c}119.3- \\
154.5\end{array}$ \\
\hline & $\begin{array}{l}\text { Max } \\
\text { Gap }\end{array}$ & 12.99 & 21.50 & 30.39 & 39.78 & 42.20 \\
\hline \multirow{2}{*}{ go } & $\begin{array}{c}\operatorname{Max} \\
T\end{array}$ & $\begin{array}{l}110.8- \\
112.8\end{array}$ & $\begin{array}{l}112.4- \\
119.2\end{array}$ & $\begin{array}{c}114- \\
123.9\end{array}$ & $\begin{array}{c}115.6- \\
128.7\end{array}$ & $\begin{array}{l}117.1- \\
133.6\end{array}$ \\
\hline & $\begin{array}{l}\text { Max } \\
\text { Gap }\end{array}$ & 11.84 & 17 & 21.47 & 25.3 & 29.1 \\
\hline \multirow[t]{2}{*}{ ijpeg } & $\begin{array}{c}\operatorname{Max} \\
\mathrm{T}\end{array}$ & $\begin{array}{l}111.7- \\
112.5\end{array}$ & $\begin{array}{c}114.2- \\
121.1\end{array}$ & $\begin{array}{c}116.8- \\
129.2\end{array}$ & $\begin{array}{l}119.3- \\
136.6\end{array}$ & $\begin{array}{l}121.5- \\
142.1\end{array}$ \\
\hline & $\begin{array}{l}\text { Max } \\
\text { Gap }\end{array}$ & 11.8 & 19 & 25.47 & 30.67 & 34.2 \\
\hline
\end{tabular}

Table 7: Maximum temperatures and temperature gaps among components for different clocks under clock gating. The maximum temperatures are shown as a range where the lower bound and the upper bound are given by the universal mode and individual mode, respectively.

than others. To reduce the temperature gaps, we propose to evenly distribute instructions to functional units. Our experiments show that temperature gaps between integer units can be virtually eliminated by this scheduling.

Table 7 presents the maximum temperatures and maximum temperature gaps with clock gating for different clocks. Compared with Table 6, the maximum temperatures with clock gating are reduced due to energy reduction but the maximum temperature gaps are increased due to dynamic throttling.

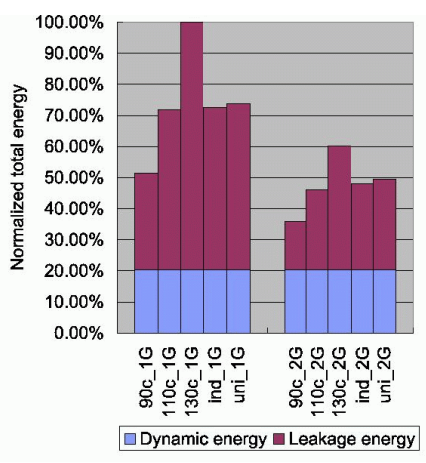

Figure 5: Energy consumption with clock gating. The conditions are the same as those in Figure 4.

\subsubsection{Energy}

Figure 5 shows the total energy consumption with clock gating for different clock. When the temperature change from $90^{\circ} \mathrm{C}$ to $130^{\circ} \mathrm{C}$, the total energy is increased by up to a factor of $2 X$ due to that the dominate leakage energy changes dramatically. From Figure 5 we can also see that leakage energy with temperature dependence in both individual mode and universal mode is close to that with fixed temperature of $110^{\circ} \mathrm{C}$, while in Figure 4, the corresponding leakage energy with temperature dependence is close to that with fixed temperature of $130^{\circ} \mathrm{C}$. The leakage energy with clock gating in Figure 5 is smaller than that without clock 
gating in Figure 4 and the overall temperature profile with clock gating is also lower as shown in Table 7. All these results again show that any study regarding to leakage energy should consider the thermal issues for accuracy.

\subsubsection{Maximum Clock}

Clock gating has no direct effect on leakage energy reduction. However, as clock gating reduces the dynamic power consumption and the system temperatures, the temperature dependent leakage power is reduced. Furthermore, the maximum clock can be increase under the same thermal constraints. Faster clock rates reduce the execution time, and therefore reduce the total leakage energy.

We use the upper bound of the maximum temperature in Table 7 as the maximum temperature. By satisfying the same maximum temperature and maximum temperature gap constraints as those in Section 4.2, Table 8 summarizes the max clocks and total leakage energy under clock gating. It is shown that we can increase the maximum clock by up to a factor of $2 X$, and reduce the total energy to as low as $51.94 \%$. In other words, the total energy can be reduced by up to $48.06 \%$.

\begin{tabular}{|c|c|c|}
\hline Benchmark & Max Clock & Leakage energy \\
\hline equake & $1 \mathrm{GHz}$ & $100 \%$ \\
\hline go & $2 \mathrm{GHz}$ & $51.94 \%$ \\
\hline ijpeg & $1.5 \mathrm{GHz}$ & $69.89 \%$ \\
\hline
\end{tabular}

Table 8: Maximum clock and corresponding normalized total energy under clock gating. The total leakage energy without clock gating under $1 \mathrm{GHz}$ clock is assumed as $100 \%$.

\section{CONCLUSIONS AND DISCUSSIONS}

Considering cycle accurate simulation, we have presented dynamic and leakage power models with clock and temperature scaling, and developed the coupled thermal and power simulation at the microarchitecture level. With this simulator, we have shown that the leakage energy and total energy can be different by up to $2.5 X$ and $2 X$ for different temperatures, respectively. Hence, microarchitecture level power simulation is hardly accurate without considering temperature dependent leakage model. We have also discussed temperature scaling of the reduced leakage power in the power-gated circuit. Such scaling model can be applied to microarchitecture level power gating presented in $[4,13]$.

As pointed out by the reviewers, a per-component thermal model similar to our individual mode was developed in [14] and was used for dynamic thermal management. The temperature calculation in [14] is essentially same as (8) and (9) in this paper. Furthermore, it was shown in [14] that the horizontal heat transfer is negligible, same as assumed in our individual mode. However, leakage power was not taken into account in [14].

In this paper we assume that the dynamic power, i.e., the switching energy per cycle is independent of the clock rate. To increase the clock rate, the designer may have to increase the supply voltage to meet the delay constraints determined by the clock rate. Dynamic voltage scaling (DVS) [15] has been proposed to consider the relationship between supply voltage and clock rate. Because the dynamic power depends on the supply voltage, our future work will consider not only leakage power scaling but also dynamic power scaling with respect to clock. We do not consider interconnect power explicitly and assume fixed floorplan in this paper. In the future, we intend to study microarchitecture power/thermal management with simultaneous interconnect power estimation and floorplanning optimization.

\section{REFERENCES}

[1] W.Ye, N.Vijaykrishnan, M.Kandemir, and M.J.Irwin, "The design and use of simplepower: a cycle-accurate energy estimation tool," in $D A C, 2000$.

[2] D.Brooks, V.Tiwari, and M.Martonosi, "Wattch: A framework for architectural-level power analysis optimization," in ISCA, 2000.

[3] A. Dhodapkar, C. Lim, and G. Cai, "Tem2p2est: A thermal enabled multi-model power/performance estimator," in Workshop on Power Aware Computer Systems, Nov 2000.

[4] W. Liao, J. M. Basile, and L. He, "Leakage power modeling and reduction with data retention," in ICCAD 02, Nov 2002.

[5] D. Sylvester and H. Kaul, "Future performance challenges in nanometer design," in $D A C, 2001$.

[6] R. Viswanath, V. Wakharkar, A. Watew, and V. Lbonheur, "Thermal performance challenges from silicon to systems," Intel Technology Journal, vol. 3, 2000.

[7] J. Butts and G. Sohi, "A static power model for architects," in Proc. of MICRO33, December 2000.

[8] D. Duarte, Y. Tsai, N. Vijaykrishnan, and M. J. Irwin, "Evaluating run-time techniques for leakage power reduction techniques," in ASP-DAC, 2002.

[9] W. Jiang, V. Tiwari, E. de la Iglesia, and A. Sinha, "Topological analysis for leakage prediction on digital circuits," in Proceedings of the 7th Asia and South Pacific Design Automation Conference, joint with the 15th International Conference on VLSI Design, 2002.

[10] J. V. D. Vegte., Feedback Control System, 3rd Edition. Prentice Hall, 1994.

[11] H. Hanson, M. Hrishikesh, V. Agarwal, S. Keckler, and D. Burger, "Static energy reduction techniques for microprocessor caches," in Proceedings of the International Conference on Computer Design, 2001.

[12] V. Tiwari, D. Singh, S. Rajgopal, and G. Mehta, "Reducing power in high-performance microprocessors," in DAC, 1998.

[13] L. Li, I. Kadayif, Y.-F. Tsai, N. Vijaykrishnan, M. Kandemir, M. J. Irwin, and A. Sivasubramaniam, "Leakage energy management in cache hierarchies," in Proceedings of International Conference on Parallel Architectures and Compilation Techniques, 2002.

[14] T. A. K. Skadron and M. Stan, "Control-theoretic techniques and thermal-rc modeling for accurate and localized dynamic thermal management," in Proceedings of the Eighth International Symposium on High-Performance Computer Architercture, 2002.

[15] T. Burd, T. Pering, A. Stratakos, and R. Bordersen, "A dynamic voltage-scaled microprocessor system," in 2000 IEEE International Solid-State Circuits Conference Digest of Technical Papers, Feb 2000. 